\title{
Single dose piperacillin in treating uncomplicated gonococcal urethritis in men
}

\author{
T THIRUMOORTHY, ${ }^{*}$ K B LIM,* C T LEE, ${ }^{*}$ E H SNG $\dagger$ \\ From *Middle Road Hospital, and the †Department of Pathology, Singapore General Hospital, Singapore
}

SUMMARY A single intramuscular dose of piperacillin $2 \mathrm{~g}$, with probenecid $1 \mathrm{~g}$ orally, was used to treat 82 men with uncomplicated gonococcal urethritis due to penicillinase producing strains of Neisseria gonorrhoeae (PPNG) or non-PPNG. All 49 patients infected with non-PPNG strains were cured, as opposed to only $25(76 \%)$ of 33 patients infected with PPNG strains, giving an overall success rate of $90 \%$. No serious side effects of treatment were observed. Post gonococcal urethritis (PGU) occurred in $18 \%$ of the patients treated. Antibiotic susceptibility tests showed that $44(96 \%)$ of the 46 non-PPNG strains tested had MICs of piperacillin of $2 \mathrm{mg} / \mathrm{l}$ or less and $26(76 \%)$ of the 34 PPNG strains had MICs of $32 \mathrm{mg} / \mathrm{l}$ or more. This regimen is not recommended for use as first line treatment in areas where there is a high incidence of infection with PPNG strains. It is, however, highly effective against non-PPNG strains.

Piperacillin is a semisynthetic broad range penicillin with good activity against Gram negative bacteria, including Neisseria gonorrhoeae, and is reported to be stable to penicillinase produced by penicillinase producing strains of $N$ gonorrhoeae (PPNG). Intramuscular piperacillin $2 \mathrm{~g}$ gives a peak serum concentration of $30 \mathrm{mg} / 1$ at 30 minutes, with an elimination half life of 81 minutes. The addition of probenecid prolongs the half life and increases the average serum concentration, both by about one third. About two thirds of the administered dose is eliminated unchanged in the urine after 24 hours. ${ }^{1}$

Wong et al tested eight PPNG strains isolated in 1981 and 1982 from South East Asia and found that $75 \%$ were inhibited by less than $0 \cdot 125 \mathrm{mg} / 1$ and $100 \%$ by less than $0.25 \mathrm{mg} / 1$ piperacillin. ${ }^{2}$ The serum concentrations attained after $2 \mathrm{~g}$ piperacillin is given intramuscularly are therefore far greater than the reported minimum inhibitory concentrations (MICs) for PPNG strains, which suggested that this drug might be useful for treating gonorrhoea in this region. We accordingly conducted this trial to evaluate the efficacy of piperacillin in treating uncomplicated gonococcal urethritis due to either PPNG or nonPPNG strains.

Address for reprints: Dr T Thirumoorthy, Middle Road Hospital, 250 Middle Road, Singapore 0718

Accepted for publication 25 January 1987

\section{Patients and methods}

STUDY POPULATION

Men attending the outpatient clinics of the Middle Road Hospital in June to August 1985 were selected if they had signs and symptoms of gonococcal urethritis and if urethral Gram stained smears showed intracellular Gram negative diplococci. Patients with a history of allergy to penicillin, evidence of coexisting syphilis, or who had received antibiotics within the preceding two weeks were excluded. Patients whose pretreatment cultures failed to grow $N$ gonorrhoeae and those who failed to return for the first follow up visit were excluded. Informed consent was obtained from alt-patients.

ISOLATION OF $N$ GONORRHOEAE AND ANTIBIOTIC SUSCEPTIBILITY TESTING

Urethral specimens obtained with sterile platinum loops were immediately inoculated on to modified Thayer-Martin medium, incubated at $36^{\circ} \mathrm{C}$ in an atmosphere of carbon dioxide, and examined after 24 to 48 hours. Gonococcal isolates were identified by colonial morphology, oxidase reaction, and Gram stained microscopic appearance. Confirmed isolates were tested for penicillinase production by the paper acidometric method as described by Sng et al. ${ }^{3}$ The minimum inhibitory concentration (MIC) of piperacillin was assessed by the agar plate dilution method described previously. ${ }^{4}$ Piperacillin was used in doubling dilutions from $2 \mathrm{mg} / \mathrm{l}$ to $128 \mathrm{mg} / \mathrm{l}$. 
TREATMENT AND FOLLOW UP

All patients were treated with a single intramuscular injection of $2 \mathrm{~g}$ piperacillin sodium into a gluteal muscle, and oral probenecid $1 \mathrm{~g}$ was given at the same time. As is standard procedure in this department, all patients receiving parenteral penicillins were observed for half an hour after the injection.

All patients were asked to return three to five days and 10 to 20 days after treatment. At each visit the patients were asked about re-exposure and examined, and urethral material for smear microscopy and cultures for $N$ gonorrhoeae was taken. Cure was established at the first follow up visit if symptoms and signs of gonococcal infection or complications were absent and smears and cultures for $N$ gonorrhoeae were negative. Patients who returned for the second follow up visit were also assessed for post gonococcal urethritis (PGU). PGU was diagnosed by the presence of five or more polymorphonuclear leucocytes per $1000 \times$ microscopic field without intracellular Gram negative diplococci and a negative culture for $N$ gonorrhoeae. All patients who attended the follow up visits were evaluated for side effects of treatment. As a clinic routine, the Venereal Disease Research Laboratory (VDRL) test was performed before treatment and six weeks and three months after treatment.

\section{Results}

\section{STUDY POPULATION}

A total of 91 men were enrolled into the study. Nine were excluded from efficacy analysis - one because of re-exposure before the first follow up visit, one because of coexisting early latent syphilis requiring treatment, one because he yielded a negative pretreatment culture, and six because they did not attend follow up. There therefore remained 82 patients aged 18 to 59 (mean 25.5) for efficacy analysis. Sixty five patients were assessed for PGU.

\section{ERADICATION OF $N$ GONORRHOEAE}

Table 1 shows the results of treatment. Only 25 (76\%) of 33 patients infected with PPNG strains were cured compared with all 49 infected with non-PPNG strains. The overall cure rate was $90 \%$.

Table 1 Response to treatment of 82 men with gonococcal urethritis

\begin{tabular}{llll}
\hline Strain & No assessed & No $(\%)$ cured & No $(\%)$ failed \\
\hline PPNG & 33 & $25(76)$ & $8(24)$ \\
Non-PPNG & 49 & $49(100)$ & 0 \\
Total & 82 & $74(90)$ & $8(10)$ \\
\hline
\end{tabular}

Table 2 Minimum inhibitory concentrations (MICs) of piperacillin for 80 strains* of Neisseria gonorrhoeae

\begin{tabular}{|c|c|c|c|c|c|c|c|}
\hline \multirow[b]{2}{*}{ Strain } & \multicolumn{7}{|c|}{ MIC $(\mathrm{mg} / \mathrm{l})$ of: } \\
\hline & $\leqslant 2$ & 4 & 8 & 16 & 32 & 64 & $\geqslant 128$ \\
\hline $\begin{array}{l}\text { PPNG }(n=34) \\
\text { Non-PPNG }(n=46)\end{array}$ & $\begin{array}{r}2 \\
44\end{array}$ & $\begin{array}{l}2 \\
2\end{array}$ & 1 & 3 & 3 & 6 & 17 \\
\hline
\end{tabular}

*Two strains were not recovered from stock cultures for MIC assessment.

\section{PGU AND SIDE EFFECTS}

PGU was diagnosed in $17(26 \%)$ of the 65 patients assessed. When asked specifically, $59(72 \%)$ patients complained of pain at the injection site, but all said the pain was bearable. No other side effects of treatment were observed.

\section{ANTIBIOTIC SUSCEPTIBILITY TESTS}

Table 2 shows the results of the antibiotic susceptibility tests. PPNG strains were very much less susceptible to piperacillin compared with non-PPNG strains; $76 \%(26 / 34)$ of the PPNG strains had MICs of $32 \mathrm{mg} / \mathrm{l}$ or more and $96 \%(44 / 46)$ of the nonPPNG strains had MICs of $2 \mathrm{mg} / 1$ or less.

\section{Discussion}

Clinical and bacteriological cure was established in $100 \%$ of 49 patients infected with non-PPNG strains as opposed to only $25(76 \%)$ of 33 infected with PPNG strains. The $100 \%$ cure rate obtained in treating non-PPNG infected patients accords well with the results of two previous studies. ${ }^{56}$ The rather low cure rate obtained in treating patients infected with PPNG strains, however, suggests that piperacillin is subjected to some hydrolysis by penicillinase from PPNG strains. This finding is also suported by the results of antibiotic susceptibility tests, which showed that PPNG strains had very high MICs of piperacillin. The results of our antibiotic susceptiblity tests are very different from those reported by Wong et al. ${ }^{2}$ They found that all eight PPNG strains isolated from South East Asia had MICs of between 0.03 and 0.25 $\mathrm{mg} / \mathrm{l}$. There has only been one other study of the efficacy of the same regimen in PPNG infections, and it reported a similar cure rate of $78 \%$ (Lancaster DJ, et $a l$, unpublished observation). No serious side effects of treatment were observed. PGU was diagnosed in $26 \%$ of patients assessed.

We conclude that the newer semisynthetic penicillin, piperacillin, though better than penicillin in range and activity, has not been shown to be as clinically active as the newer cephalosporins against PPNG 
strains. Intramuscular piperacillin $2 \mathrm{~g}$ plus oral probenecid $1 \mathrm{~g}$ is, however, very effective for gonorrhoea due to non-PPNG strains. It may find a favourable place where procaine penicillin $4.8 \mathrm{MIU}$ is used as first line treatment.

We thank Cyanamid Far East for supplying piperacillin for this study and Dr Tulip Tan, Medical Director, for her encouragment. We also thank YL Koh, WL Yeo, the other nurses in charge of the male outpatient department, and all the medical and laboratory staff for their co-operation.

\section{References}

1 Tjandramaga TB, Mullie A, Verbesselt R, DeSchepper PJ, Verbist L. Piperacillin: human pharmacokinetics after intra- venous and intramuscular administration. Antimicrob Agents Chemother 1978;14:829-37.

2 Wong PCL, Wing YH, Ng WWS. In-vitro stability of B-lactam antibiotics to hydrolysis by B-lactamases of Nesseria gonorrhoeae from South East Asia. Sex Transm Dis 1985;12:128-34.

3 Sng EH, Yeo KL, Rajan VS. Simple method for detecting penicillinase-producing Neisseria gonorrhoeae and Staphylococcus aureus. British Journal of Venereal Diseases 1981;57: 141-2.

4 Sng EH, Rajan VS, Pang R, Yeo KL. Susceptibility of Neisseria gonorrhoeae to cefotaxime and ceftizoxime. British Journal of Venereal Diseases 1981;57:162-4.

5 Landis SJ, Ramphal R, Mansheim BJ, Rand KH, Shands JW. Comparative efficacy of piperacillin and penicillin $G$ in the treatment of gonococcal urethritis. Antimicrob Agents Chemother 1981;20:693-5.

6 Simpson ML, Khan MY, Siddigui Y, Gruninger RP, Wigren DI. Comparison of piperacillin and penicillin in the treatment of uncomplicated gonorrhoea. Antimicrob Agents Chemother 1982;21:727-9. 